\title{
Association between epicardial adipose tissue thickness and coronary heart disease: A meta- analysis
}

\begin{abstract}
Objective: To systematically evaluate the efficacy and diagnostic value of epicardial adipose tissue in coronary artery disease.
\end{abstract}

\begin{abstract}
Methods: The authors searched PubMed, Embase, Web of Science, CNKI and CBM from the database to January 2019, and collected articles on the clinical trials of epicardial adipose tissue in coronary heart disease. The improved Jadad scale was used for quality evaluation, and Revman 5.3 software was used for metaanalysis of the included literature.

Results: Finally, 21 articles were included, including a total of 4049 patients. EAT was $12.212 .62 \mathrm{~mm}$ in the CAD group and $9.921 .37 \mathrm{~mm}$ in the non-CAD group. EAT was $2.221 .86 \mathrm{~mm}$ in the CAD group and $1.81 .4 \mathrm{~mm}$ in the non-CAD group. The random effect model was adopted for the study $\left(I^{2}=95 \%, p<0.00001, Q=398.94\right.$, $\mathrm{df}[\mathrm{Q}]=20$ ), and heterogeneity was found in all cases. The EAT of CAD patients was significantly higher than that of non-CAD patients $(S M D=1.07,95 \% \mathrm{Cl}=0.75-1.38, \mathrm{p}<0.00001$ ). EAT of CAD patients in echocardiography group and CT measurement group was higher than that of non-CAD patients.
\end{abstract}

Conclusion: According to the results of this meta-analysis, EAT in patients undergoing echocardiography or CT examination has certain value in the diagnosis and risk stratification of coronary artery disease due to its ease-of-use, cost-effectiveness and non-exposure characteristics, and is worthy of further clinical exploration.

Keywords: Epicardial adipose tissue - Epicardial fat - Coronary artery disease - Atherosclerosis

\section{Introduction}

Epicardial adipose tissue (EAT) is located in the visceral fat around the heart, derived from embryonic development [1], and is the brown adipose tissue around the coronary arteries, epicardium and peritoneal fat under the epicardium. As an endocrine organ, EAT is inseparable from metabolic syndrome [2]. As an important risk factor for cardiac metabolism in past studies have confirmed [3], due to the close to the anatomy of the heart, and lack of fascia boundary, EAT and paracrine or vascular secretion pathway, be play an important role in the process of atherosclerosis of hormone and fat formation factor, endocrine function, adjust the coronary artery and myocardial [4]. Recently, as a new indicator to evaluate the development of atherosclerosis, it has been paid more and more attention. Studies have found that the epicardial adipose tissue is associated with a variety of cardiac metabolic risk factors such as serum low-density lipoprotein cholesterol, fasting insulin, plasma adiponectin, blood glucose and systolic/diastolic blood pressure [5-7]. Blood pressure and visceral adipose tissue play an important role in atherosclerosis of coronary heart disease. In addition, previous studies have shown that EAT may affect the progression of coronary atherosclerosis as an inflammatory organ [8], so EAT may be correlated with the diagnosis and risk stratification of coronary heart disease, which is of clinical significance. In this paper, the published literatures were summarized and meta analyzed in order to provide references for clinical studies. Here's the report.

\section{Materials and Methods}

Research identification and selection of inclusion criteria

1. Subjects: Patients with coronary heart disease or cardiovascular disease;
Yuan Shuai ${ }^{1,2}$, Ai Ke-

Qing ${ }^{1,2}$, Yang Xin-Quan ${ }^{3}$, Xiong

Ting $^{3}$, Zhang Rong ${ }^{2}$, Han Xiao ${ }^{1,2}$ Li Jia-Jing 1,2, Zhang Shu-Meng ${ }^{1,2}$, Zhao Fei-Fei ${ }^{2}$, Wang Fang ${ }^{1,2}$, Xiao Yuan-Yuan 1,2, Gong YuNan 1,2, Lu Kai1,2, Liu Yuan ${ }^{1,2}$, Li $\mathrm{Qi}^{1,2}$, Ren Yan-Xia ${ }^{1,2}$, Qian Zhi$\mathrm{Min}^{1,2}$, Sun Chao ${ }^{1,2}$, Liang JiaYang ${ }^{1,2}$, Xu Bao ${ }^{1,2}$, Zhang Zhu' ${ }^{1,2}$, Yin Hui-Jiao 1,2 , Li Xiao-Du ${ }^{1,2}$, Mao Yu-Lin'2, Huang Liu-Hai ${ }^{1,2}$, Yue Jian ${ }^{1,2}$, He Sheng-Hu ${ }^{2}$ and Wang Da-Xin²*

'Graduate School of Dalian Medical University, Dalian, P.R. China

${ }^{2}$ Department of Cardiology, North Jiangsu People's Hospital, Yangzhou University, Yangzhou, P.R. China

${ }^{3}$ Xiangya Medical College, Central South University, Changsha, Hunan, P.R. China

*Author for correspondence: Email: daxinw2016@126.com Received date: April 18, 2019 Accepted date: April 29, 2019 Published date: May 06, 2019 
2. Intervention measures: The experimental group was patients with coronary heart disease or cardiovascular disease;

3. No language restrictions

4. Measurement tools: Echocardiography or CT

5. Study more than 20 patients

6. CT and ultrasound are only two monitoring methods, which are not the focus of this paper. The thickness of the pericardial adipose tissue in the included study was investigated, and we recorded and analyzed the differences in patients with and without coronary heart disease [9-18].

\section{Exclusion criteria}

1. Comment articles

2. Inconsistency between grouping and intervention measures

3. Loss of follow-up rate $20 \%$;

4. Case report

5. Failure to report EFT mean and standard deviation between the two groups

6. No coronary heart disease related reports (the "literature not reporting CAD" is too broad, and there are too many contributing factors. Not included in this article). Data extraction and quality assessment data were extracted independently by two authors, including: title, first author name, year of publication, underlying disease, sample size, intervention, literature tools, and data of test results.

\section{Retrieval strategy}

The databases including MEDLINE/PubMed, Embase, Web of Science, CNKI, and CBM were searched, and the database was established until March 31, 2019. Keywords: "epicardial adipose tissue, epicardial fat pad, subepicardial adipose tissue, periepicardial tissue, subepicardial adipose tissue, coronary artery disease, diagnostic techniques and procedures, myocardial ischemia, myocardial infarction, coronary angiography; Acute coronary syndrome, angina pectoris, stabilization, noninvasive methods (elastography), coronary stenosis. Abstracts and titles of articles found in the database are reviewed by two different reviewers to exclude articles that are not relevant to the content. If the studies excluded from each database were different, the differences were examined for similar judgments, and references to all relevant articles were reviewed and added. Grey literature was not evaluated in this study [19-35].

\section{Literature quality evaluation}

Use the Jadad score to assess the quality of selected studies. Four cases of random sequence generation, randomization, blind method, exit or exit were recorded in the literature. The first 3 methods are correct 2 points, do not know 1 point, inappropriate 0 points, 1 point, the fourth description and sinus 0 points. Low quality literature 3, high quality scores 4 .

In this meta-analysis, no study had a significant impact on the overall estimation, no publication bias was found in the study, and no missing studies were found. Therefore, the results of this meta-analysis are of high universality. On the other hand, we qualitatively evaluate articles using valid checklists (Figure 1).

\section{Analysis results and summary}

Meta-analysis was performed using RevMan 5.3 from the Cochrane collaboration. Standard mean difference (SMD) and 95\% confidence interval (CI) of EAT between CAD and non-CAD groups were calculated. Heterogeneity was analyzed by chi-square test and quantified by $\mathrm{I}^{2}$ index, $\mathrm{p}<0.05$ was considered statistically significant.

\section{Results}

\section{Literature screening}

A total of 552 references were initially searched, and experimental studies with reading problems and abstracts not meeting the requirements were excluded. Finally, 21 controlled clinical studies were included. The basic information and quality evaluation results of this study are shown in Figure 2.

\section{Characteristics of literature research}

This meta-analysis included a study of 21 studies involving 4,049 patients, including 2,573 patients with coronary heart disease and 1,476 patients in the control group (1,622 with CAD and 814 without CAD). In most studies $(n=10)$, coronary heart disease is considered to have more than $50 \%$ of primary coronary artery stenosis; Included in the study, angiography was considered a gold standard, the diagnosis of CAD in most studies ( $\mathrm{n}=15)$ EAT using Echocardiography:

1. The ultrasonic probe located in sternum beside the long shaft section, end-diastolic freeze image, sampling line perpendicular to the right ventricular free wall, place the aortic ring, positioned near the sternum short shaft section (papillary muscle level); 

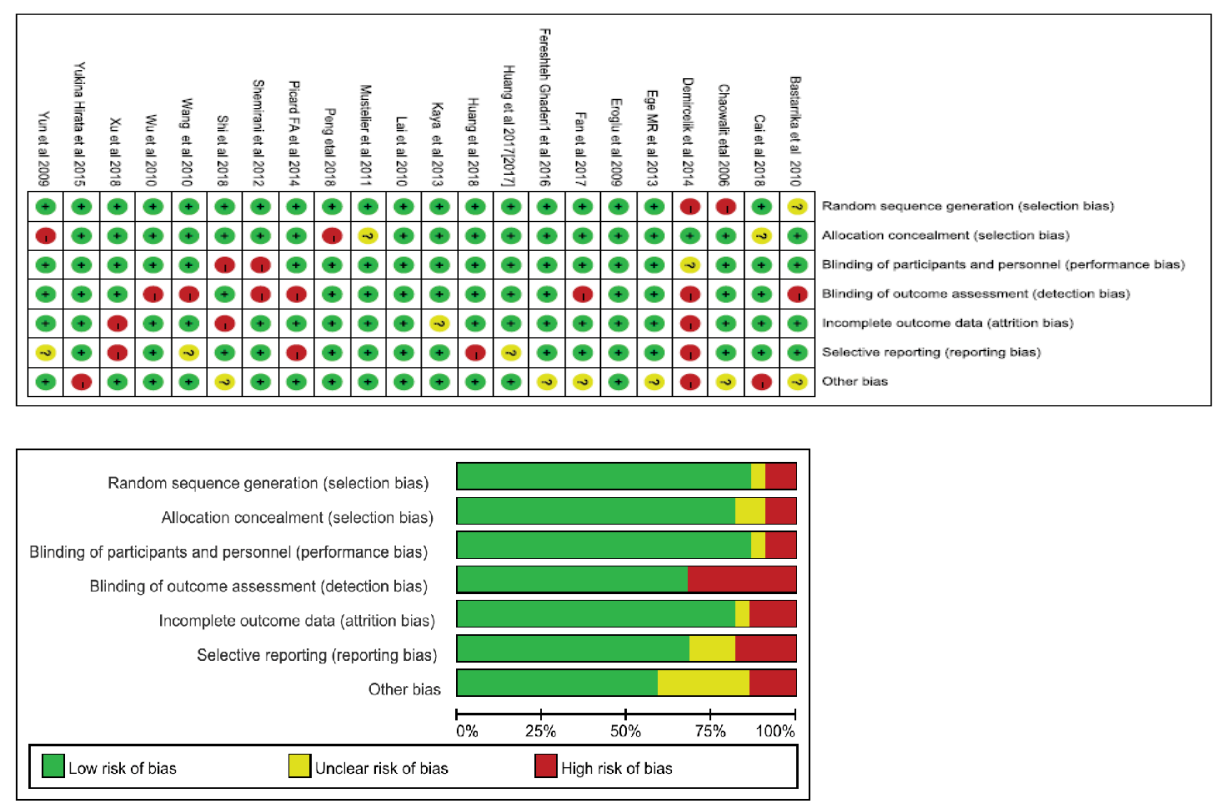

Figure 1: Summary table of publication bias included in the study.
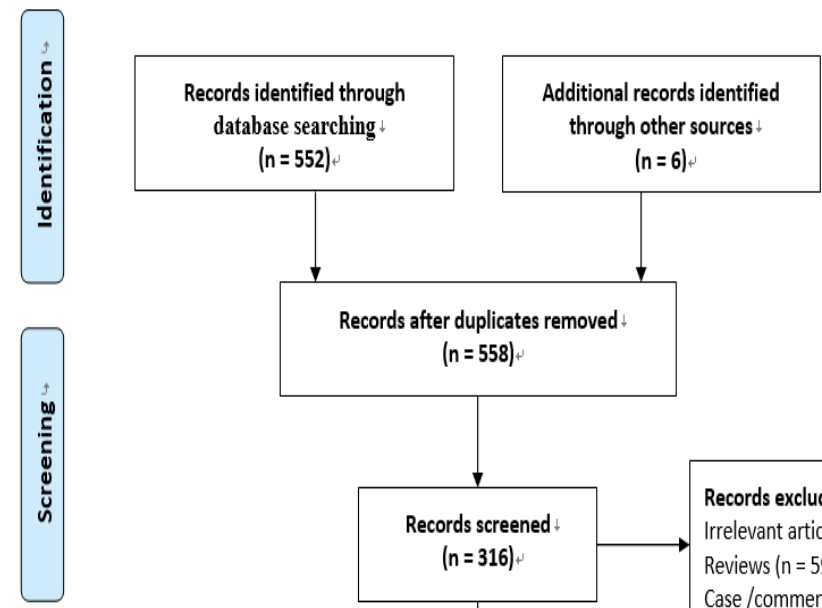

Records after duplicates removed $(n=558)$
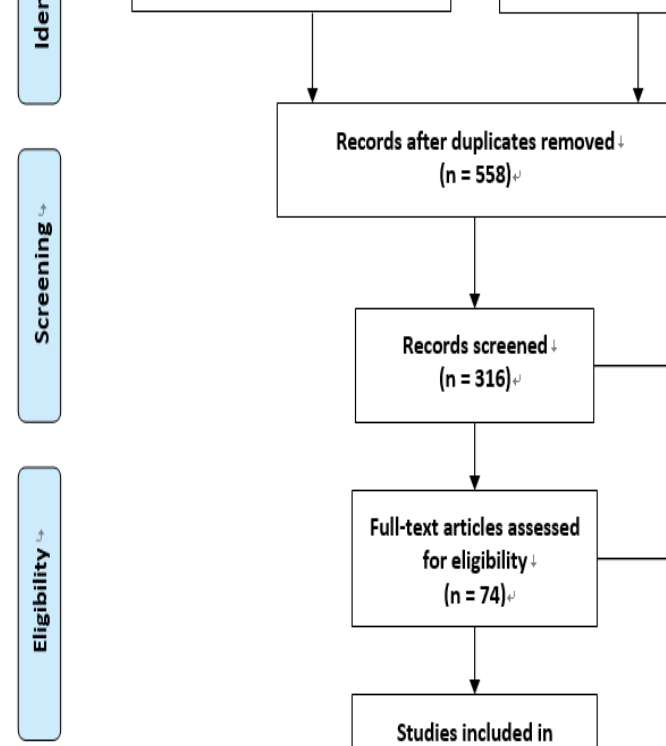

Records excluded $(n=242)$

Irrelevant articles $(n=164)$

Reviews $(n=59)$

Case /comment/letter $(n=19)$

Records excluded $(n=53)$

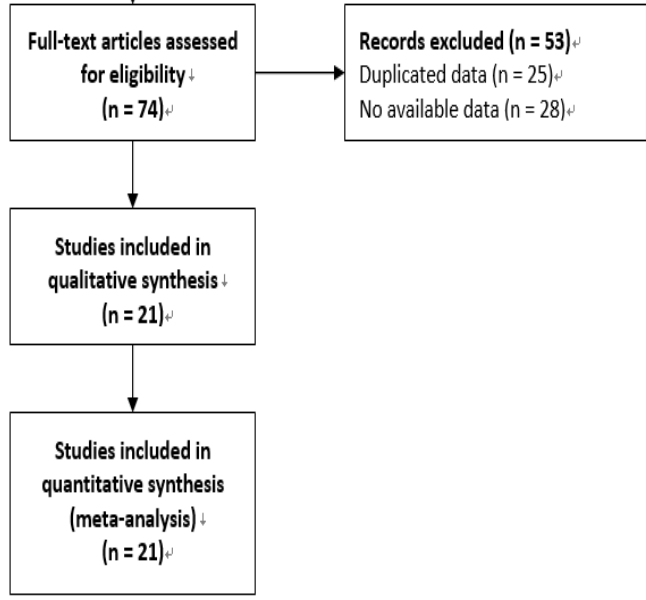

Figure 2: Meta analysis based on PRISMA statement included in the study flow chart. 
2. Also at the end of diastolic freeze image, the sample line was perpendicular to the ventricular septum, and the thickness of epicardial fat between the right ventricular free wall and the pericardial visceral layer was measured. A small number of studies $(n=6)$ have involved Computed Tomography. The minimum sample size was 45 cases and the maximum sample size was 970 cases. The first was published in 2006 and the latest in 2018 .

\section{Meta-analysis results}

Before the study, the literature that did not meet the research conditions was excluded to ensure the quality of the literature, and the sensitivity analysis of a single index was carried out. The results showed that there was no significant change in the results of the fixed-effect model and the random-effect model, that is, the Metaanalysis results of this study were stable and reliable. The random-effect model was used for the study $\left(\mathrm{I}^{2}=95 \%\right.$, $\mathrm{p}<0.00001, \mathrm{Q}=398.94$, df $[\mathrm{Q}]=20)$. The thickness of EAT in CAD patients was significantly higher than that of non-CAD patients $(\mathrm{SMD}=1.07 ; 95 \% \mathrm{CI}=0.75-1.38$, $\mathrm{p}<0.00001)$. And echocardiography measurement EAT (SMD $=1.00,95 \% \mathrm{CI}=0.62-1.38, \mathrm{p}<0.00001)$; CT measurement EFT (SMD $=1.00,95 \% \mathrm{CI}=0.70$ $1.30, \mathrm{p}<0.00001)$. For each study and overall analysis of Epicardial Fat Thickness (EFT) in patients with and without Coronary Artery Disease (CAD) (Figures 3-5) and Table1.

\section{Discussion}

Recently, coronary heart disease has become the most important factor leading to death. Since the epicardial fat is adjacent to the myocardium and coronary arteries, it may be more closely related to coronary artery disease. Eroglu et al. [17] found that compared with patients with normal coronary arteries, the EAT thickness of CAD patients increased significantly. EAT was also thicker in patients with severe CAD compared to those

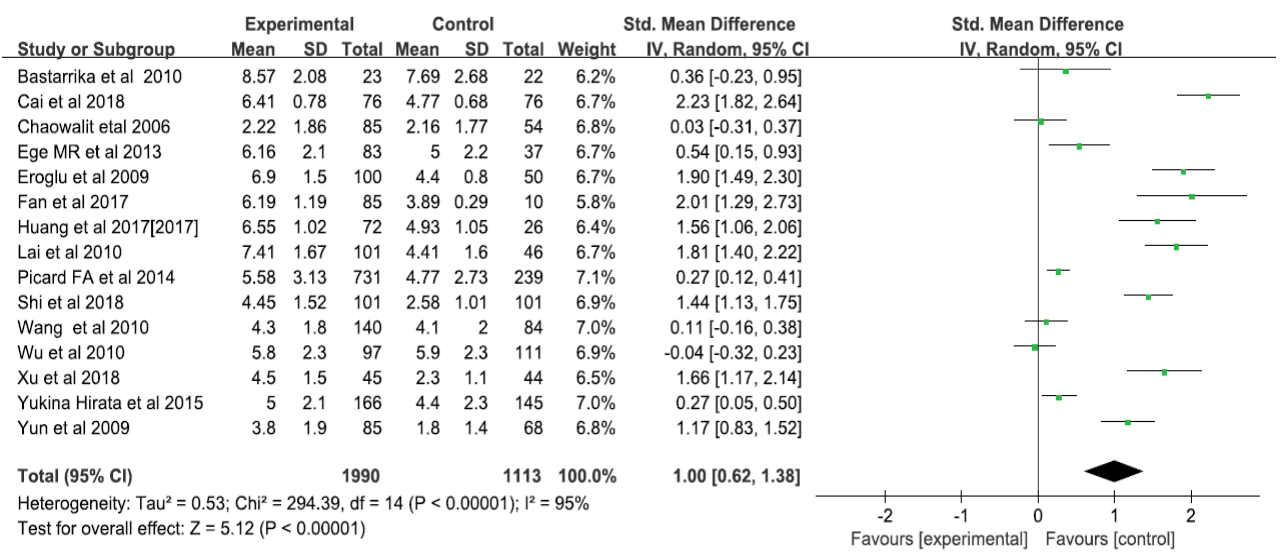

Figure 3: EAT and CAD (Mean and standard deviation of epicardial adipose thickness (measured by echocardiography, CT) in patients with and without Coronary Artery Disease (CAD) in each study and overall analysis).

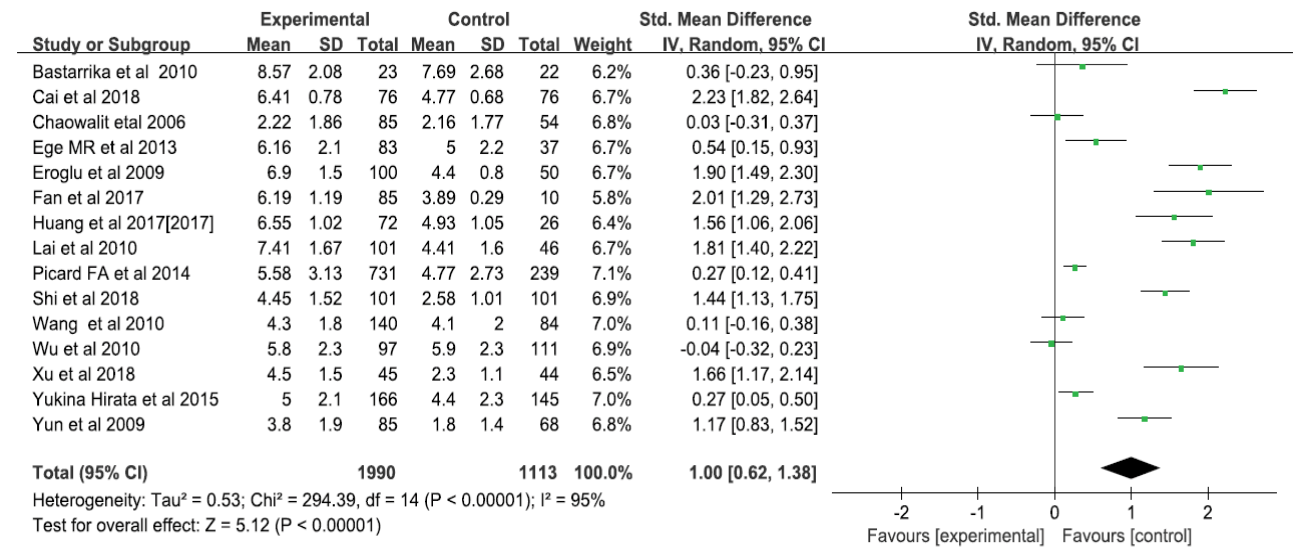

Figure 4: EAT and CAD (Mean and standard deviation of epicardial adipose thickness (measured by echocardiography) in patients with and without Coronary Artery Disease (CAD) in each study and overall analysis). 


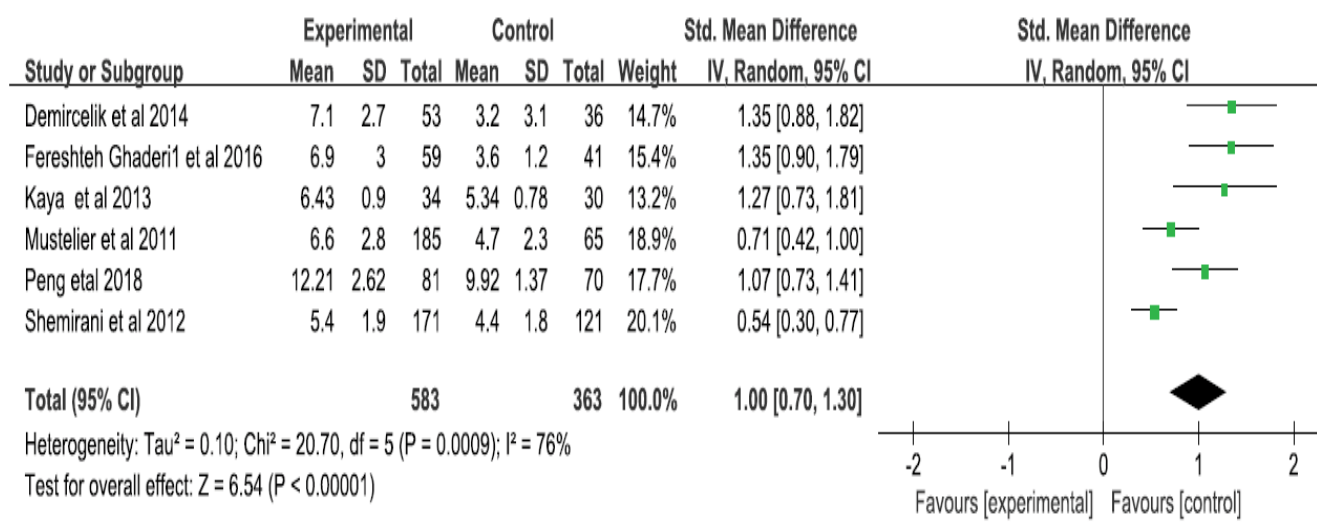

Figure 5: EAT and CAD (Mean and standard deviation of epicardial adipose thickness (measured by CT) in patients with and without Coronary Artery Disease (CAD) in each study and overall analysis).

Table 1: Meta analysis of epicardial fat thickness (EFT), coronary heart disease (CAD) and non-coronary heart disease (CAD) patients included in this study.

\begin{tabular}{|c|c|c|c|c|c|c|}
\hline \multirow{2}{*}{ Included in the study } & $\mathbf{N}$ & $\mathbf{C A D}$ & $\mathbf{n}$ & \multicolumn{2}{|c|}{ Non-CAD } & Tools to \\
\cline { 2 - 6 } & (total) & $\mathbf{n}$ & $\begin{array}{c}\text { EAT Thickness/ } \\
\mathbf{m m}\end{array}$ & $\mathbf{n}$ & EAT Thickness/mm & Measurement \\
\hline Yukina Hirata et al [9] & 311 & 166 & $5 \pm 2.1$ & 145 & $4.4 \pm 2.3$ & Echocardiography \\
\hline Fereshteh Ghaderi1 et al [10] & 100 & 59 & $6.9 \pm 3$ & 41 & $3.6 \pm 1.2$ & Computed Tomography \\
\hline Demircelik et al [11] & 89 & 53 & $7.1 \pm 2.7$ & 36 & $3.2 \pm 3.1$ & Computed Tomography \\
\hline Ege MR et al [12] & 120 & 83 & $6.16 \pm 2.1$ & 37 & $5 \pm 2.2$ & Echocardiography \\
\hline Kaya et al [13] & 64 & 34 & $6.43 \pm 0.9$ & 30 & $5.34 \pm 0.78$ & Computed Tomography \\
\hline Shemirani et al [14] & 292 & 171 & $5.4 \pm 1.9$ & 121 & $4.4 \pm 1.8$ & Computed Tomography \\
\hline Mustelier et al [15] & 250 & 185 & $6.6 \pm 2.8$ & 65 & $4.7 \pm 2.3$ & Computed Tomography \\
\hline Lai et al [16] & 147 & 101 & $7.41 \pm 1.67$ & 46 & $4.41 \pm 1.6$ & Echocardiography \\
\hline Wu et al [17] & 208 & 97 & $5.8 \pm 2.3$ & 111 & $5.9 \pm 2.3$ & Echocardiography \\
\hline Bastarrika et al [18] & 45 & 23 & $8.57 \pm 2.08$ & 22 & $7.69 \pm 2.68$ & Echocardiography \\
\hline Wang et al [19] & 224 & 140 & $4.3 \pm 1.8$ & 84 & $4.1 \pm 2$ & Echocardiography \\
\hline Eroglu et al [20] & 150 & 100 & $6.9 \pm 1.5$ & 50 & $4.4 \pm 0.8$ & Echocardiography \\
\hline Yun et al [21] & 153 & 85 & $3.8 \pm 1.9$ & 68 & $1.8 \pm 1.4$ & Echocardiography \\
\hline Picard FA et al [22] & 970 & 731 & $5.58 \pm 3.13$ & 239 & $4.77 \pm 2.73$ & Echocardiography \\
\hline Chaowalit et al [23] & 139 & 85 & $2.22 \pm 1.86$ & 54 & $2.16 \pm 1.77$ & Echocardiography \\
\hline Huang et al [24] & 98 & 72 & $6.55 \pm 1.02$ & 26 & $4.93 \pm 1.05$ & Echocardiography \\
\hline Cai et al [25] & 152 & 76 & $6.41 \pm 0.78$ & 76 & $4.77 \pm 0.68$ & Echocardiography \\
\hline Peng et al [26] & 151 & 81 & $12.21 \pm 2.62$ & 70 & $9.92 \pm 1.37$ & Computed Tomography \\
\hline Xu et al [27] & 89 & 45 & $4.5 \pm 1.5$ & 44 & $2.3 \pm 1.1$ & Echocardiography \\
\hline Shi et al [28] & 202 & 101 & $4.45 \pm 1.52$ & 101 & $2.58 \pm 1.01$ & Echocardiography \\
\hline Fan et al [29] & 95 & 85 & $6.19 \pm 1.19$ & 10 & $3.89 \pm 0.29$ & Echocardiography \\
\hline
\end{tabular}

with mild $\mathrm{CAD}$ and in patients with multiple diseases compared to those with single CAD, suggesting a correlation between EAT thickness and CAD severity.

EAT may promote the occurrence of coronary heart disease through mitochondrial dysfunction, NF-kB signaling pathway activation, LXR/RXR activation signaling pathway inhibition and other pathways. Recently, for example, it has also been found that changes in fat metabolism can lead to increased reactive oxygen species and stress responses [34,35].
An important feature of cardiovascular diseases is mitochondrial dysfunction, which leads to abnormal energy metabolism and increased production of ROS. Adipose tissue is a key organ that regulates energy balance and is involved in energy storage and consumption. It is also now thought of as an endocrine organ that releases various adipokines, including adipokines such as adiponectin. It interacts with other organs such as the heart and skeletal muscle. Impaired mitochondrial activity in adipocytes is usually associated with decreased oxidation of fatty acids, resulting in increased free fatty 
acids in the cytoplasm, leading to deterioration of organ function. Mitochondria are highly dynamic organelles that undergo morphological changes, including fusion, division, and regulatory turnover. Mitochondrial dysfunction includes the reduction of mitochondrial content, the change of mitochondrial morphology, the reduction of electron transport chain complex activity, the opening of mitochondrial permeability transition hole, the increase of ROS production and other different characteristics. The formation of excessive ROS will have harmful effects on proteins and lipids, leading to cell dysfunction and eventually cell death. In cardiomyocytes, ROS are produced in different regions by different enzymes, including NADPH oxidase on the cell membrane and xanthine oxidase in the cytoplasm. However, mitochondria are the most important cellular source of ROS. The proximity of mitochondrial DNA to ROS production sites and the lack of protection of mitochondrial DNA by histones make mitochondrial DNA (mt DNA) highly susceptible to oxidative stress, thus promoting the development of cardiovascular diseases.

The intrinsic inflammatory response to EAT also contributes to the progression of atherosclerosis. Under physiological conditions, proinflammatory and anti-inflammatory adipokines secreted by EAT act on myocardium and coronary arteries through paracrine and vasocrine pathways. Generally speaking, the two are in a relatively balanced state and have a protective effect on the heart. However, when the body is in a chronic low-grade inflammatory state for a long time, the balance between proinflammatory and antiinflammatory adipokines secreted by EAT is broken, and the expression of anti-inflammatory adipokines is down-regulated. The expression of NF- B, JNK and TLR was significantly increased in patients with coronary heart disease. Activation of TLR induces translocation of NF- B into the nucleus, leading to increased release of pro-atherogenic inflammatory factors such as il-1, il6, TNF and resistin with deleterious effects on adjacent myocardium and coronary arteries. A study on the mRNA expression of chemerin in CAD patients' EAT found that the expression of chemerin was up-regulated and adiponectin was down-regulated in CAD patients' EAT. Therefore, chemerin enhanced macrophage migration and foam cell formation, while the inhibition of foam cell transformation by adiponectin and macrophage coagulation was weakened. This suggests that the imbalance between anti-inflammatory and proinflammatory adipokines can affect the function of macrophages and eventually lead to Acute Coronary Syndrome (ACS).

Therefore, early diagnosis and prediction of lifethreatening organ-specific manifestations are necessary to improve the prognosis of patients with coronary heart disease. Recent studies have shown that EAT is significantly associated with early identification of coronary heart disease, but the rate of missed diagnosis is high. It is of great clinical significance to evaluate the predictive value of epicardial adipose tissue for the occurrence, development and severity of coronary artery disease [30]. The results of this study showed that the CAD patients had significantly higher EAT than the non-CAD group. Although many studies have revealed the relationship between EAT and CAD, the reliable evidence is limited. Some studies have shown that there is a significant relationship between EAT and dyslipidemia. Dyslipidemia is considered as one of the known risk factors for atherosclerosis, so EAT is indeed associated with coronary heart disease. In addition, a study shows epicardial fat with a wide range of the expression of inflammatory factors such as the CCL2, IL - $1 \mathrm{~b}$, interleukin 6 , and TNF-a, heart disease patients release of high levels of phospholipase A2 type II secretion, thus facilitating the production of inflammatory medium of lipid, phospholipase A2 type II secretion of high level and increased risk of coronary artery disease (CAD) independent factors [33-35]. It is worth further study and evaluation.

Obesity and obesity-related metabolic and cardiovascular diseases, particularly coronary heart disease, continue to increase globally, creating a huge burden of disease. With the deepening of studies, researchers have gradually realized that in addition to reducing Visceral adipose tissue (visceral adipose tissue, VAT) accumulation, it is also very important to regulate the function of adipose tissue, especially to adjust the chronic low-degree inflammatory state caused by longterm imbalance of EAT pro-inflammatory and antiinflammatory adipokines, which may be a new target for the treatment of coronary heart disease.

\section{Conclusion}

Over the years, a series of novel pro-inflammatory and anti-inflammatory adipokines expressed and secreted by EAT that are associated with coronary heart disease have been discovered, but their role in coronary heart disease has not been fully demonstrated. Therefore, 
it is necessary to further explore the interaction and regulation mechanism between EAT and coronary atherosclerosis, further deepen the understanding of

\section{References}

1. Hirata Y. Clinical utility of measuring epicardial adipose tissue thickness with echocardiography using a high-frequency linear probe in patients with coronary artery disease. $J$ Am Soc Echocardiogr. 28(10): 1240-1246 (2015).

2. Yorgun $\mathrm{H}$. Increased epicardial fat tissue is a marker of metabolic syndrome in adult patients. Int J Cardiol. 165(2): 308-313 (2013).

3. Zhang M. Correlation between epicardial adipose tissue and coronary flow reserve in coronary heart disease patients with no chest pain. Beijing Da. 46(6): 848-853 (2014).

4. Luna-Luna M. Differential expression of osteopontin, and osteoprotegerin mRNA in epicardial adipose tissue between patients with severe coronary artery disease and aortic valvular stenosis: Association with HDL subclasses. Lipids Health Dis. 16(1): 156 (2017).

5. Tok D. Increased epicardial fat thickness is associated with low grade systemic inflammation in metabolic syndrome. Turk Kardiyol Dern Ars. 40(8): 690-695 (2012).

6. Drapkina J, Drapkina O. Epicardial fat as the predictor for essential hypertension and early vascular damage in patients with metabolic syndrome and non-alcoholic fatty liver disease. Endosc Ultrasound. 3: S2 (2014).

7. Ege MR, Guray U. Serum gamma-glutamyltransferase levels correlate with epicardial adipose tissue thickness in patients with coronary artery disease. Angiol. 64(1): 21-25 (2013).

8. Diaz-Rodriguez E. Effects of dapagliflozin on human epicardial adipose tissue: Modulation of insulin resistance, inflammatory chemokine production, and differentiation ability. Cardiovasc Res. 114(2): 336-346 (2018).

9. Ghaderi F. Assosiation of epicardial and pericardial fat thickness with coronary artery disease. Electron Physician. 8(9): 2982-2989 (2016).

10. Demircelik MB. Epicardial adipose tissue and pericoronary fat thickness measured with 64-multidetector computed tomography: potential predictors of the severity of coronary artery disease. Clinics (Sao Paulo). 69(6): 388-392 (2014).

11. Kaya H. Relation of epicardial fat thickness and brachial flowmediated vasodilation with coronary artery disease. J Cardiol. 62(6): 343-347 (2013).

12. Shemirani H, Khoshavi M. Correlation of echocardiographic epicardial fat thickness with severity of coronary artery diseasean observational study. Anadolu Kardiyol Derg. 12(3): 200-205 (2012).

13. Cabrera-Rego JO. Echocardiographic measurement of epicardial fat thickness: in search for a consensus/Correlation of echocardiographic epicardial fat thickness with severity of coronary artery disease-an observational study. Anadolu Kardiyol Derg. 12(5): 448-449 (2012).

14. Wu FZ. Impact of location of epicardial adipose tissue, measured by coronary artery calcium-scoring computed tomography on obstructive coronary artery disease. Am J Cardiol. 112(7): 943949 (2013). obesity, adipokines and coronary heart disease, and search for effective treatment strategies for coronary artery disease.

15. Bastarrika G. Relationship between coronary artery disease and epicardial adipose tissue quantification at cardiac CT: comparison between automatic volumetric measurement and manual bidimensional estimation. Acad Radiol. 17(6): 727-734 (2010).

16. Wang TD. Association of epicardial adipose tissue with coronary atherosclerosis is region-specific and independent of conventional risk factors and intra-abdominal adiposity. Atherosclerosis. 213(1): 279-287 (2010).

17. Eroglu S. Epicardial adipose tissue thickness by echocardiography is a marker for the presence and severity of coronary artery disease. Nutr Metab Cardiovasc Dis. 19(3): 211-217 (2009).

18. Yun KH. Relationship between the Echocardiographic Epicardial Adipose Tissue Thickness and Serum Adiponectin in Patients with Angina. J Cardiovasc Ultrasound. 17(4): 121-126 (2009).

19. Picard FA. Epicardial adipose tissue thickness correlates with the presence and severity of angiographic coronary artery disease in stable patients with chest pain. PLoS One. 9(10): e110005 (2014).

20. Chaowalit N. Subepicardial adipose tissue and the presence and severity of coronary artery disease. Atherosclerosis. 186(2): 354359 (2006).

21. Xiaoyun H. Ultrasound study on the correlation between thickness of epithelial adipose tissue and coronary artery disease. Chinese J eviden-based cardiovasc med. 9(09): 1102-1104 (2017).

22. Weibiao CAI. Predictive value of epicardial adipose tissue thickness for coronary heart disease. J Rare Dis. 25(04): 17-24 (2018).

23. Peng P. The relationship between coronary artery peripheral epicardial fat thickness and CT value and coronary heart disease. Anbui medical university. p: 38 (2018).

24. Yalan X. A study on the correlation between the thickness of epicardial fat layer and neck vascular elasticity in patients with coronary heart disease by ultrasonography and their correlation. Clinical study. 26 (04): 138-139 (2018).

25. Haihong S. A study on the correlation between the thickness of epicardial adipose layer and neck vascular elasticity in patients with coronary heart disease by ultrasonography and their correlation. Clinical study. 26 (2): 191-192 (2018).

26. Hao F. A study on the correlation between the thickness of epicardial fat and the complexity of coronary heart disease, 2017. Xinjiang medical university. p: 43 (2018).

27. Lee KC. Is the epicardial adipose tissue area on non-ECG gated low-dose chest CT useful for predicting coronary atherosclerosis in an asymptomatic population considered for lung cancer screening? Eur Radiol. 29(2): 932-940 (2019).

28. Karastergiou K. Epicardial adipokines in obesity and coronary artery disease induce atherogenic changes in monocytes and endothelial cells. Arterioscler Thromb Vasc Biol. 30(7): 1340-1346 (2010).

29. Pierdomenico SD. Meta-analysis of the relation of 
echocardiographic epicardial adipose tissue thickness and the metabolic syndrome. Am J Cardiol. 111(1): 73-78 (2013).

30. Boengler K. Mitochondria and ageing: role in heart, skeletal muscle and adipose tissue. J Cachexia Sarcopenia Muscle. 8(3): 349-369 (2017).

31. Liu Z. Nterminal truncated peroxisome proliferator activated receptor gamma coactivatorlalpha alleviates phenylephrine induced mitochondrial dysfunction and decreases lipid droplet accumulation in neonatal rat cardiomyocytes. Mol Med Rep. 18(2): 2142-2152 (2018).

32. Hilse KE. The expression of uncoupling protein 3 coincides with the fatty acid oxidation type of metabolism in adult murine heart. Front Physiol. 9: 747 (2018).

33. Iacobellis G, Bianco AC. Epicardial adipose tissue: emerging physiological, pathophysiological and clinical features. Trends Endocrinol Metab. 22(11): 450-457 (2011).

34. Gao X. Association of chemerin mRNA expression in human epicardial adipose tissue with coronary atherosclerosis. Cardiovasc Diabetol. 10: 87 (2011).

35. Okamoto Y. Adiponectin inhibits macrophage tissue factor, a key trigger of thrombosis in disrupted atherosclerotic plaques. Atherosclerosis. 226(2): 373-377 (2013). 